INPLASY

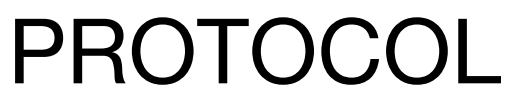

To cite: Li et al. Therapeutic effect of Xuebijing combined with thymosin on hemorrhagic fever with renal syndrome: a protocol of systematic review and meta-analysis. Inplasy protocol 202040068. doi: 10.37766/inplasy2020.4.0068

Received: 12 April 2020

Published: 12 April 2020

Corresponding author: Chun-mei Li

Chun-meiLi@outlook.com

Author Affiliation:

First Affiliated Hospital of Jiamusi University

Support: SRPHLJHFPC (2018175)

Review Stage at time of this submission: The review has not yet started.

Conflicts of interest: No.

\section{Therapeutic effect of Xuebijing combined with thymosin on hemorrhagic fever with renal syndrome: a protocol of systematic review and meta-analysis}

$\mathrm{Li}, \mathrm{CM}^{1}$; Sun, Q2.

Review question / Objective: Is Xuebijing combined with thymosin (XBJ-T) effective and safety for the treatment of patients with hemorrhagic fever with renal syndrome (HFRS)? Condition being studied: Renal syndrome; hemorrhagic fever; Xuebijing; thymosin.

Information sources: This study will search the electronic databases of Cochrane Library, PubMed, EMBASE, PsycINFO, Scopus, Opengrey, Cumulative Index to Nursing and Allied Health Literature, Web of Science, Google Scholar, Allied and Complementary Medicine Database, and Chinese Biomedical Literature Database from the beginning of each database to the present. This study will not employ any language and publication status. The example of Cochrane Library database will be built. We will also adapt similar search strategies to the other electronic databases. In addition to the electronic databases, we will also manually search other related journals and conference proceedings, as well as the reference lists of relevant reviews.

INPLASY registration number: This protocol was registered with the International Platform of Registered Systematic Review and Meta-Analysis Protocols (INPLASY) on 12 April 2020 and was last updated on 12 April 2020 (registration number INPLASY202040068).

\section{INTRODUCTION}

Review question / Objective: Is Xuebijing combined with thymosin (XBJ-T) effective and safety for the treatment of patients with hemorrhagic fever with renal syndrome (HFRS)?

Condition being studied: Renal syndrome; hemorrhagic fever; Xuebijing; thymosin. 


\section{METHODS}

Participant or population: All participants (18 years old or older) who clinically diagnosed as HFRS will be included in this study. No race, sex, and educational background will be applied.

Intervention: In the intervention group, all forms of XBJ-T regardless of dosage, treatment period will all be included in this study.

Comparator: In the control group, we will consider any managements as the comparators, but not XBJ-T.

Study designs to be included: This study will include randomized controlled trials (RCTs) on assessing the effect of XBJ-T for the treatment of patients with HFRS.

Eligibility criteria: This study will include RCTs ON assessing the effect of XBJ-T vs. other managements for the treatment of patients with HFRS.

Information sources: This study will search the electronic databases of Cochrane Library, PubMed, EMBASE, PsycINFO, Scopus, Opengrey, Cumulative Index to Nursing and Allied Health Literature, Web of Science, Google Scholar, Allied and Complementary Medicine Database, and Chinese Biomedical Literature Database from the beginning of each database to the present. This study will not employ any language and publication status. The example of Cochrane Library database will be built. We will also adapt similar search strategies to the other electronic databases. In addition to the electronic databases, we will also manually search other related journals and conference proceedings, as well as the reference lists of relevant reviews.

Main outcome(s): The primary outcomes are positive rate of specific $\operatorname{Ig} M$ and positive rate of specific IgG. They were measured by tested with colloidal gold immuno-dot assay or others.
Additional outcome(s): The secondary outcomes are rate of mortality, improving the viremia, the clinical conditions, restoring kidney function and platelet account, and occurrence rate of complications.

Data management: Two researchers will independently utilize the pre-designed data extraction sheet to collect the data. It includes data from several aspects, including general study information, patient characteristics, study setting, study methods, sample size, interventions, comparators, outcomes and other relevant indicators. If there are any inconsistencies between two researchers, a third researcher will help to resolve them by discussion. If we identify any missing data or unclear data, we will contact primary authors to request this information by email.

Quality assessment / Risk of bias analysis: Two researchers will independently assess the risk of bias for each included trial using Cochrane risk of bias tool. It covers 7 aspects and each one is determined as three levels: low, unclear, and high risk of bias. Any inconsistencies will be resolved by a third researcher through consultation.

Strategy of data synthesis: RevMan $\mathbf{5 . 3}$ software will be used to conduct data synthesis and data analysis. As for continuous outcome indicators, they will be expressed as mean difference and $95 \%$ confidence intervals (CIs). As for dichotomous outcome indicators, they will be exerted as risk ratio and $95 \%$ Cls. We will check statistical heterogeneity across included trials using $I^{2}$ test. $I^{2} \leq 50 \%$ indicates low heterogeneity, while $\mathrm{I}^{2}>50 \%$ means obvious heterogeneity. If the trials are sufficiently similar, we will pool the data from original studies using a fixed-effect model when $\mathrm{I}^{2} \leq \mathbf{5 0} \%$. On the other hand, if I $^{2}>\mathbf{5 0 \%}$, we will synthesize the data using a random-effect model. We will also carry out subgroup analysis to explore the causes of obvious heterogeneity.

Subgroup analysis: We will undertake subgroup analysis to identify the source of 
heterogeneity based on the different interventions, comparators, and outcome indicators.

Sensibility analysis: We will perform sensitivity analysis to examine the robustness of pooled results by taking away low quality studies.

Country(ies) involved: China.

Keywords: Renal syndrome; hemorrhagic fever; Xuebijing; thymosin; randomized controlled trial; effect; safety. 